\title{
On the determination of a global strain rate model
}

\author{
Corné Kreemer ${ }^{1}$, John Haines ${ }^{2}$, William E. Holt ${ }^{1}$, Geoffrey Blewitt ${ }^{3}$, and David Lavallee ${ }^{4}$ \\ ${ }^{1}$ Department of Geosciences, State University of New York at Stony Brook, U.S.A. \\ ${ }^{2}$ Bullard Laboratories, Cambridge University, U.K. \\ ${ }^{3}$ Nevada Bureau of Mines and Geology, and Seismological Laboratory, University of Nevada, Reno, U.S.A. \\ ${ }^{4}$ Department of Geomatics, University of Newcastle upon Tyne, U.K.
}

(Received December 28, 1999; Revised June 12, 2000; Accepted June 13, 2000)

\begin{abstract}
The objective of this paper is to outline the fundamental concepts underlying the estimation of a global strain rate model. We use a variant of the method first introduced by Haines and Holt (1993) to estimate the strain rate tensor field within all of the Earth's deforming regions. Currently the observables used are $\sim 1650$ geodetic velocities, seismic moment tensors from the Harvard CMT catalog, and Quaternary fault slip rate data. A model strain rate field and velocity field are obtained in a least-squares fit to both the geodetic velocities and the observed strain rates inferred from fault slip rates. Seismic moment tensors are used to provide a priori constraints on the style and direction (not magnitude) of the model strain rate field for regions where no fault slip rate data are available. The model will soon be expanded to include spreading rates, ocean transform azimuths, and more fault slip rate data. We present a first estimate of the second invariant of the global model strain rate field. We also present Euler poles obtained by fitting geodetic vectors located on defined rigid plates. We find that $17 \%$ of the total model moment rate is accommodated in zones of (diffuse) continental deformation.
\end{abstract}

\section{Introduction}

Ever since the concept of plate tectonics was established in the mid-60's, numerous studies have been performed to model plate motions (e.g., Chase, 1972; Minster and Jordan, 1978; DeMets et al., 1990). However, these models only describe motions of the rigid plates, not motions over the entire Earth. To date no direct measurements of presentday motions within diffuse zones of deformation have been included in any global velocity model. Over the last decade space geodetic techniques have provided a large quantity of high-accuracy measurements of motions on stable plates as well as within (diffuse) plate boundary zones (Fig. 1). Adopting the methodology of Haines and Holt (1993) we determine a velocity gradient tensor field for the entire Earth by performing a least-squares inversion of geodetic velocities and observed strain rates inferred from slip on Quaternary faults. This enables us to infer a global velocity model, as well as estimate a global strain rate field within zones of (diffuse) deformation.

The aim of this study is to obtain an estimate for the ongoing strain rate field that is associated with the accomodation of present-day plate motion. It has been shown in several studies that over sufficient length-scales, of the order of the entire width of a plate boundary zones, geodetic velocities are often indistinguishable from relative plate velocities (e.g., Stein, 1993; Shen-Tu et al., 1999; Kreemer et al., 2000). Lateral changes in the strain rate field due to elastic loading of the lithosphere within plate boundary zones will have the ef-

Copy right (C) The Society of Geomagnetism and Earth, Planetary and Space Sciences (SGEPSS); The Seismological Society of Japan; The Volcanological Society of Japan; The Geodetic Society of Japan; The Japanese Society for Planetary Sciences. fect of reducing our estimate of the spatial resolution of the ongoing strain rates. However, it is not the goal of this study to infer model slip rates of all individual faults. Instead we hope to infer regional strain rates and styles of deformation within fault zones with a horizontal scale of resolution of the order of 50-300 km (a factor of 1-6 times the width of our grid areas). Furthermore, we do not attempt to model temporal changes in strain rates. Therefore, to avoid inclusion of coseismic and post-seismic deformation within plate boundaries, each geodetic velocity vector is carefully considered before inclusion in the model.

A global strain rate field estimate has the potential of serving as a self-consistent global reference model for the magnitude and style of regional present-day, horizontal, crustal deformation. In this paper we describe the fundamental assumptions and methodology underlying the estimation of a global strain rate field.

\section{The Parameterization of a Horizontal Velocity Gradient Tensor Field}

When the horizontal dimensions of interest are several times the thickness of the brittle elastic layer, it is sometimes convenient to parameterize the horizontal velocity field as a continuum (e.g., England and McKenzie, 1982). The continuous velocity field can be parameterized as a function of the deformation field on the Earth's surface;

$$
\overline{\mathbf{u}}=r[\overline{\mathbf{W}}(\widehat{\mathbf{x}}) \times \widehat{\mathbf{x}}],
$$

where $\widehat{\mathbf{x}}$ is the unit radial position vector, $r$ is the Earth's radius, and $\overline{\mathbf{W}}(\widehat{\mathbf{x}})$ is the rotation vector function. Model strain rates involve spatial derivatives of the vector function $\overline{\mathbf{W}}(\widehat{\mathbf{x}})$ (Haines and Holt, 1993; Haines et al., 1998). The rotation 


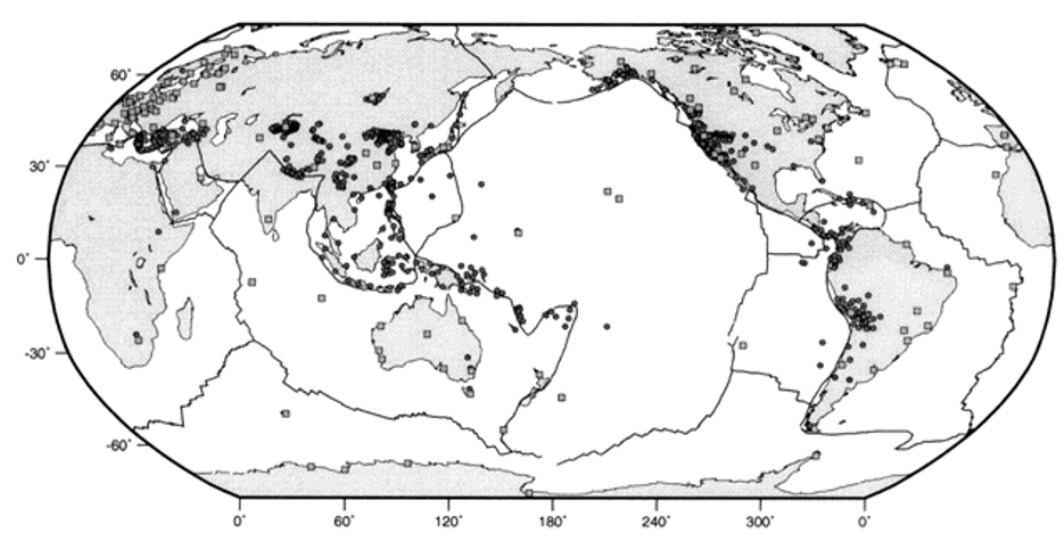

Fig. 1. Site locations of the geodetic velocities used in this study. Squares are IGS stations used in our reference frame solution, and circles are all other sites for which velocities are used (list of references can be acquired through the authors or at http://www.terrapub. co.jp/ journals/EPS/pdf/5210/append.pdf).

vector function $\overline{\mathbf{W}}(\widehat{\mathbf{x}})$ is expanded using bi-cubic Bessel interpolation (De Boor, 1978) on a curvilinear grid, allowing rapid variations in the model strain rate field (Haines et al., 1998). The distribution of $\overline{\mathbf{W}}(\widehat{\mathbf{x}})$ is determined through a least-squares minimization between observed and model velocities and observed and model strain rates within grid areas. Plate rigidity can be simulated by setting the spatial derivatives of the rotation vector function $\overline{\mathbf{W}}(\widehat{\mathbf{x}})$ to zero, such that areas that lie on the same plate have the same rotation vector function, or Euler pole. This pole can either be solved for in the inversion or can be constrained, a priori, to a known rotation pole.

Observed average seismic strain rates for any grid area can be obtained by summing moment tensors in the volume described by the product of the grid area and the assumed seismogenic thickness (Kostrov, 1974);

$$
\dot{\varepsilon}_{i j}=\frac{1}{2 \mu V T} \sum_{k=1}^{N} M_{0} m_{i j}
$$

where $N$ is the number of events in the grid area, $\mu$ is the shear modulus, $V$ the cell volume, $T$ is the time period of the earthquake record, $M_{0}$ is the seismic moment, and $m_{i j}$ is the unit moment tensor. Similarly, average horizontal strain rate components from Quaternary fault slip data are obtained by a variant of Kostrov's (1974) summation;

$$
\dot{\varepsilon}_{i j}=\frac{1}{2} \sum_{k=1}^{n} \frac{L_{k} \dot{u}_{k}}{A \sin \delta_{k}} m_{i j}^{k},
$$

where $m_{i j}$ is the unit moment tensor defined by the fault orientation and unit slip vector, and $n$ is the number of fault segments in grid area $A$, each having a length $L_{k}$, dip angle $\delta_{k}$, and slip rate $\dot{u}_{k}$.

The objective function that is minimized in the least squares inversion is

$$
\begin{array}{r}
\sum_{1}^{N}\left(\dot{\varepsilon}_{i j}^{\mathrm{fit}}-\dot{\varepsilon}_{i j}^{\mathrm{obs}}\right)\left(\dot{\varepsilon}_{p q}^{\mathrm{fit}}-\dot{\varepsilon}_{p q}^{\mathrm{obs}}\right)(V)_{i j, p q}^{-1}+ \\
\sum_{1}^{M}\left(u_{i}^{\mathrm{fit}}-u_{i}^{\mathrm{obs}}\right)\left(u_{j}^{\mathrm{fit}}-u_{j}^{\mathrm{obs}}\right) C_{i j}^{-1}
\end{array}
$$

where $i j$, and $p q$ denote tensor components of the strain rate tensor, $\dot{\varepsilon}_{i j}^{\text {obs }}$ is the average strain rate inferred for each area using the Kostrov summation, $\dot{\varepsilon}_{i j}^{\text {it }}$ is the average value of the model strain rate for the given area, defined by the continuous spline functions, $V$ is the a priori variance-covariance matrix of the inferred strain rates, and $N$ is the number of grid areas. The second part of the objective function involves the observed velocities $\mathbf{u}^{\text {obs }}$, which are to be fitted by the model velocity field (Eq. (1)) in a defined frame of reference. Tensor $C_{i j}$ contains the variance-covariance values of the observed geodetic velocity vectors, and $M$ is the number of velocity observations.

\subsection{Constraints on the style of deformation}

For regions that are not densely sampled with geodetic observations, the interpolation of geodetic velocities can be highly non-unique in describing the regional strain rate field (Kreemer et al., 2000; Beavan and Haines, 2000). However, the design of the strain rate covariance matrix can place some a priori constraints on the model strain rate field. The minimum set of constraints involves the assignment of isotropic strain rate variances. When variances are isotropic the model strain rate field is free to deform in any direction in order to fit observed velocity vectors. The assigned variances vary globally in magnitude such that plate boundaries that accommodate high relative motions are allowed to strain at higher rates in the process of fitting observed velocities (Haines et al., 1998; Kreemer et al., 2000; Beavan and Haines, 2000).

Strain rate covariances can be made anisotropic such that the model strain rate field matches the expected directions of the two horizontal principal axes, inferred from the seismic strain field, to within a specified confidence level. The expected directions of principal strain are determined for each grid area using moment tensors of all shallow earthquakes $(\leq 40 \mathrm{~km})$ in the Harvard centroid moment tensor (CMT) catalog. In the estimation of the expected directions of principal strain rate all earthquake mechanisms are weighted equally in Kostrov's (1974) summation. We emphasize that the constraint on the direction of the model principal strain rate not only involves an uncertainty of $\pm 10^{\circ}$, but also that in the minimization of the objective function (Eq. (4)), priority is given to an optimum fit to the geodetic velocities. 
Covariances can also be constructed such that the model strain rate field matches the style of strain rate (dip-slip, strike-slip, or mixed) inferred from seismic strain rates (detailed formulation is given in Haines et al. (1998)). This procedure is adopted in this paper in areas where there are no Quaternary fault slip rate data. However, if earthquake activity is low or absent, covariances are made isotropic. It is important to note that only the direction and relative magnitude of the principal axes of strain rate are constrained, not the sign of strain rate (i.e., no a priori differentiation is made between convergence or divergence, and left or right lateral shear). The magnitude of the model strain rate field is not inferred using the seismic data (For applications of this methodology see Shen-Tu et al. (1998) and Kreemer et al. (2000)).

Slip rate estimates on Quaternary faults provide the best constraint on the magnitude, style, and localization of the expected strain rates, especially for areas where the geodetic observations are sparse (Shen-Tu et al., 1999; Holt et al., 2000). We use Quaternary fault slip rates compiled within central and east Asia (England and Molnar, 1997). For areas where geodetic velocities are densely observed, the geodetic strain rates may not always match the spatial variations in geologic strain rates. For example, this is the case in southern California where the San Andreas fault is locked near the big bend and strain rates inferred from geodetic observations are distributed in comparison with geologic rates (Shen-Tu et al., 1999). However, over a maximum length scale of 200-300 $\mathrm{km}$, the integrated rates of strain inferred from Quaternary fault slip rates yield velocities indistinguishable from those directly inferred from geodetic measurements (Shen-Tu et al., 1999).

Along ocean boundaries extensive data sets are available with the strikes of transforms and ridges and with spreading rate data (DeMets et al., 1990; Spitzak and DeMets, 1996). We will eventually incorporate spreading rates and transform and ridge azimuths as velocity constraints.

\section{Model Grid and Reference Frames}

Our model grid is continuous in longitudinal direction and covers most deforming areas between $80^{\circ} \mathrm{N}$ and $80^{\circ} \mathrm{S}$. Each grid area is $0.6^{\circ}$ by $0.5^{\circ}$ in dimension. Whether an area is considered to be deforming or not is based primarily on seismicity occurrence (Engdahl et al. (1998) and the CMT catalog). All oceanic ridge and transform zones are allowed to deform and thus accomodate relative plate motion. Within oceanic and continental regions of diffuse deformation, where seismicity rates are often low, the designation of boundaries between deforming and plate-like regions was often subjective. Therefore, the geometry of deforming regions in this model should be viewed as approximate. Currently $\sim 28000$ grid areas cover the Earth's deforming regions; all other areas are considered to be rigid. The rigid areas mimic 22 independent plates and blocks, including a number of relatively small entities such as the Rivera plate, Anatolian plate, Ochotsk plate, Caroline plate, among others.

One of the main advantages of the methodology described in this paper is that an unlimited amount of different geodetic studies can be combined. The original reference frame of each individual study does not have to be adopted and the reference frame can be determined in the inversion; the reference frames for each study are solved for in the process of finding one self-consistent velocity gradient tensor field. That is, implicit in our procedure is the assumption that a single rigid body rotation can be applied to vectors from each individual study (one rotation vector for each study) such that the rotated vectors will provide a "best fit" to the model velocity field. The single most important geodetic information is given by data provided by the International GPS Service (IGS), because IGS stations are located on all major plates. Moreover, most regional geodetic studies are tied to major plates, hence an accurate estimate of present-day plate motions is important. In the next section we describe how we obtain our 'reference frame solution' using IGS data.

\section{The Reference Frame Solution}

Our reference frame solution is a combination of weekly global and regional Global Positioning System (GPS) solutions for 127 stations from the IGS Densification Project (Zumberge and Liu, 1995) between 1995 and 1999. Weekly station coordinate estimates from the 7 global IGS analysis centers and 3 regional associate analysis centers (Australia, Europe, and South America) are rigorously combined using a free-network approach by the Newcastle Global Network Analysis Centre (Davies and Blewitt, 2000). A modified Helmert blocking approach is taken utilizing stochastic modeling to minimize frame bias. Weekly evolving variance component estimates, antenna height corrections and a three dimensional data-snooping outlier rejection method are also applied.

All station constraints are removed prior to combination and the variance and covariance matrices are augmented to remove arbitrary rotation constraints. Any stations appearing in a minimum of 65 weekly solutions are fitted to a constant linear station model applying minimal constraints for network orientation and orientation rate. An iterative residual outlier rejection boundary of 6 standard deviations is used to exclude outliers in each station residual series (mainly the non-global stations). The resulting free network solution is aligned to ITRF97 by estimating and applying a 12 parameter Helmert transformation (no scale or scale rate); this infers a geocenter from satellite laser ranging; i.e., free from GPS orbit modeling.

\section{Results to Date}

In the model to date $\sim 1650$ geodetic velocities are used from 42 different studies, containing GPS, Satellite Laser Ranging (SLR), and Very Long Baseline Interferometry (VLBI) techniques (Fig. 1). A self-consistent global velocity and strain rate field has been obtained in the process of fitting these velocities in combination with observed strain rates inferred from Quaternary fault slip rates (England and Molnar, 1997). For areas where no fault slip rate data are available constraints are applied on the style and direction of the model strain rate tensor field from the seismic strain tensor field inferred from all shallow events in the CMT catalog (January 1977-October 1999).

In our model we find a weighted RMS of 0.52 for the fit of the model velocities with the geodetic velocities. Table 1 contains estimated Euler poles for relative plate 
Table 1. Relative angular velocities as determined in this study.

\begin{tabular}{|c|c|c|c|c|c|c|c|c|c|}
\hline \multirow{2}{*}{$\begin{array}{c}\text { Plate pair } \\
\text { EU-NA }\end{array}$} & \multicolumn{3}{|c|}{ This study } & \multicolumn{3}{|c|}{ Larson et al. (1997) } & \multicolumn{3}{|c|}{ DeMets et al. (1994) } \\
\hline & 64.9 & 127.3 & 0.23 & 68.1 & 126.6 & 0.24 & 62.4 & 135.8 & 0.21 \\
\hline PA-NA & -50.3 & 100.7 & 0.76 & -49.6 & 95.7 & 0.83 & -48.7 & 101.8 & 0.75 \\
\hline SA-NA & -22.3 & 131.9 & 0.15 & -11.1 & 126.7 & 0.29 & -16.4 & 121.9 & 0.15 \\
\hline NZ-SA & 45.2 & -91.3 & 0.57 & 43.8 & -84.8 & 0.74 & 56.1 & -94.0 & 0.72 \\
\hline AN-SA & 85.4 & 105.8 & 0.23 & 63.8 & -53.5 & 0.24 & 86.4 & -40.7 & 0.26 \\
\hline AN-PA & 65.0 & -91.5 & 0.84 & 63.6 & -95.0 & 0.93 & 64.5 & -84.0 & 0.87 \\
\hline AU-PA & 64.4 & 9.9 & 0.95 & 65.7 & 2.9 & 1.04 & 60.2 & 1.7 & 1.07 \\
\hline NZ-PA & 54.0 & -94.2 & 0.99 & 52.5 & -94.5 & 1.37 & 55.8 & -90.1 & 1.36 \\
\hline AU-EU & 8.8 & 50.7 & 0.62 & 8.6 & 48.5 & 0.65 & 15.2 & 40.5 & 0.69 \\
\hline AU-AN & 12.0 & 44.9 & 0.62 & 9.8 & 43.2 & 0.65 & 13.2 & 38.8 & 0.65 \\
\hline NZ-AN & 22.2 & -90.5 & 0.45 & 30.0 & -94.0 & 0.49 & 40.7 & -95.9 & 0.52 \\
\hline CO-NA & 13.5 & -96.6 & 1.40 & & - & & 27.9 & -120.7 & 1.36 \\
\hline CO-PA & 26.5 & -92.1 & 2.05 & & - & & 36.8 & -108.6 & 2.00 \\
\hline PH-PA & -1.3 & -43.9 & 0.96 & & - & & -1.2 & -45.8 & 0.96 \\
\hline IN-EU & 27.0 & 12.7 & 0.35 & & - & & 24.4 & 17.7 & 0.51 \\
\hline AR-EU & 23.0 & 7.9 & 0.26 & & - & & 24.6 & 13.7 & 0.50 \\
\hline PH-EU & -49.1 & -21.8 & 1.08 & & - & & -48.1 & -23.2 & 1.04 \\
\hline
\end{tabular}

Angular velocities are for the first plate relative to the second. Poles are given by latitude $\left({ }^{\circ} \mathrm{N}\right)$, longitude $\left({ }^{\circ} \mathrm{E}\right)$, and angular rate $\left({ }^{\circ} \mathrm{Myr}{ }^{-1}\right)$. Plate abbreviations: EU, Eurasia; NA, North America; PA, Pacific; SA, South America; NZ, Nazca; AN, Antarctica; AU, Australia; CO, Cocos; PH, Philippine Sea; IN, India; AR, Arabia.

motion of some plate pairs. Table 1 also shows Euler pole estimates from a global GPS study using IGS velocities (Larson et al., 1997) and from the NUVEL-1A plate motion model (DeMets et al., 1994). Angular rotation vectors (plus their covariance matrices) for all plates can be found at http://www.terrapub.co.jp/journals/EPS/ pdf/5210/append.pdf. The table on this website also contains, for each geodetic study, the angular velocities (plus covariance matrices) that were obtained in the inversion and applied to each study to rotate vectors into a global selfconsistent reference frame. In general we find a good agreement between our model results and NUVEL-1A and, especially, Larson et al. (1997), but also some notable differences exist. Some of these differences are discussed here. We find that the rotation vector for the North America (NA) plate relative to the Pacific plate (PA) is located $5^{\circ}$ more to the east than Larson et al. (1997) found. We believe that this difference may be related to Larson et al.'s (1997) inclusion of the velocity at Fairbanks into their calculation of the rotation vector of NA. When we include Fairbanks on the stable NA plate the weighted RMS for the IGS sites that we use on stable NA increases from 0.11 to 0.27 . Therefore, we prefer to consider Fairbanks inside the PA-NA plate boundary zone. The rotation rate for the Nazca plate relative to the South America and Pacific plates is significantly lower in this study than in most previous studies. This is in agreement with what is found in recent geodetic studies (Angermann et al., 1999; Norabuena et al., 1999). We find rotation rates for the Arabian and In- dian plates relative to Eurasia that are $48 \%$ and $31 \%$ slower than NUVEL-1A, respectively. To our knowledge this is the first estimate made for the Arabia-Eurasia rotation vector using geodetic data. The relatively low rotation rate of India with respect to Eurasia implies a velocity of $35 \mathrm{~mm} \mathrm{yr}^{-1}$ at Bangalore, which is $\sim 13 \mathrm{~mm} \mathrm{yr}^{-1}$ slower than NUVEL-1A and $7-12 \mathrm{~mm} \mathrm{yr}^{-1}$ slower than earlier geodetic studies (e.g., Shen et al., 2000). We attribute this decrepancy to the fact that the model velocity at this site prefers to fit our geodetic velocity inferred from IGS data ( $36 \mathrm{~mm} \mathrm{yr}^{-1}$ ) above fitting the geodetic velocities from other studies, which all indicate higher velocities at Bangalore.

Because our strain rate model includes only fault slip information for the central and east Asia region and because it contains no information from oceanic spreading rates and transform azimuths, estimates of the model strain rate field are still subject to significant uncertainties in style, magnitude, and localization. However, on a global scale a reasonably accurate model can be obtained with the present data set. We present the second invariant of the model strain rate tensor field in Fig. 2. We estimate the tectonic moment rate from the model strain rate field and we assume a globally constant shear modulus of $3.5 \times 10^{10} \mathrm{Nm}^{-2}$ and a seismogenic depth of $20 \mathrm{~km}$ for continental zones, $30 \mathrm{~km}$ for subduction zones, $20 \mathrm{~km}$ for zones of diffuse oceanic deformation, and $7.5 \mathrm{~km}$ for oceanic ridges and transforms. Initial calculations from the preliminary model indicate a total tectonic moment rate of $7.7 \times 10^{21} \mathrm{Nm} \mathrm{yr}^{-1}$ within the shallow seismogenic 


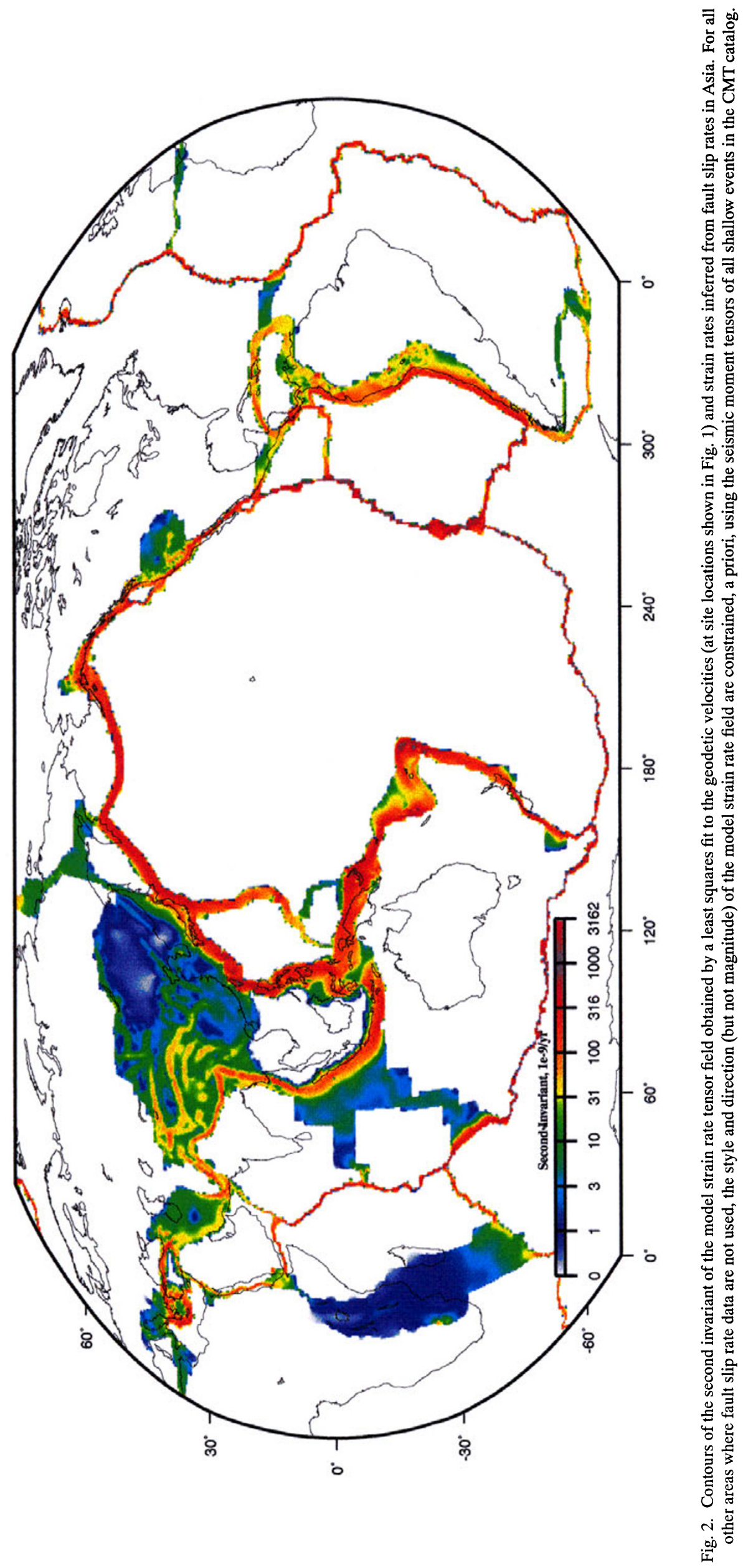


portion of the Earth's lithosphere. Moment rates within areas of (diffuse) continental deformation add up to $1.3 \times 10^{21}$ $\mathrm{Nm} \mathrm{yr}^{-1}$, which is $17 \%$ of the global tectonic moment rate.

Acknowledgments. We thank Boudewijn Ambrosius, Rick Bennett, Jeff Freymueller, Brad Hager, and Teruyuki Kato for providing unpublished GPS results. We also thank all members of the IGS community and in particular the analysis centres; Australian Surveying and Land Information Group (AUSLIG), Center for Orbit Determination Europe (CODE), European Coordination Regional Network Analysis Centre (EUREF), European Space Operations Center (ESOC), GeoForschungsZentrum (GFZ), Jet Propulsion Laboratory (JPL), National Oceanic and Atmospheric Administration (NOAA), Natural Resources Canada (NRCAN), Scripps Institution of Oceanography (SIO) and The IGS Regional Network Associate Analysis Centre for South America (SIRGAS) for making their weekly solutions available. This paper benefited from constructive reviews by Shimon Wdowinski and Kosuke Heki. W.E.H is funded by NSF grant EAR-9628872 and NASA grant SENH990325-0015. G.B. is funded by the same NASA grant through a subcontract with SUNY. D.L. is funded by a Natural Environment Research Council (UK) studentship.

\section{References}

Angermann, D., J. Klotz, and C. Reigber, Space-geodetic estimation of the Nazca-South America Euler vector, Earth Platet. Sci. Lett., 171, 329-334, 1999.

Chase, C. G., The n-plate problem of plate tectonics, Geophys. J. R. Astron. Soc., 29, 117-122, 1972

Beavan, J. and J. Haines, Contemporary horizontal velocity and strain rate fields of the Pacific-Australian plate boundary zone through New Zealand, J. Geophys. Res., 2000 (in press).

Davies, P. and G. Blewitt, Methodology for global geodetic time series estimation: a new tool for geodynamics, J. Geophys. Res., 105, 11,083$11,100,2000$

De Boor, C., A Practical Guide to Splines, p. 392, Springer-Verlag, New York, 1978.

DeMets, C., R. G. Gordon, D. F. Argus, and S. Stein, Current plate motions, Geophys. J. Int., 101, 425-478, 1990.

DeMets, C., R. G. Gordon, D. F. Argus, and S. Stein, Effect of recent revisions of the geomagnetic reversal time scale on estimates of current plate motions, Geophys. Res. Lett., 21, 2191-2194, 1994.

Engdahl, E. R., R. van der Hilst, and R. Buland, Global teleseismic relocation with improved travel times and procedures for depth determination, Bull. Seismol. Soc. Am., 88, 722-743, 1998.

England, P. C. and D. P. McKenzie, A thin viscous sheet model for continental deformation, Geophys. J. R. Astron. Soc., 70, 295-321, 1982.

England, P. C. and P. Molnar, The field of crustal velocity in Asia calculated from Quaternary rates of slip on faults, Geophys. J. Int., 130, 551-582, 1997.

Haines, A. J. and W. E. Holt, A procedure for obtaining the complete horizontal motions within zones of distributed deformation from the inversion of strain rate data, J. Geophys. Res., 98, 12,057-12,082, 1993.

Haines, A. J., J. A. Jackson, W. E. Holt, and D. C. Agnew, Representing distributed deformation by continuous velocity fields, Sci. Rept. 98/5, Inst. of Geol. and Nucl. Sci., Wellington, New Zealand, 1998.

Holt, W. E., N. Chamot-Rooke, X. LePichon, A. J. Haines, B. Shen-Tu, and J. Ren, The velocity field in Asia inferred from Quaternary fault slip rates and GPS observations, J. Geophys. Res., 2000 (in press).

Kostrov, V. V., Seismic moment and energy of earthquakes, and seismic flow of rocks, Izv. Acad. Sci. USSR Phys. Solid Earth, 1, Eng. Transl., 23-44, 1974.

Kreemer, C., W. E. Holt, S. Goes, and R. Govers, Active deformation in eastern Indonesia and the Philippines from GPS and seismicity data, $J$. Geophys. Res., 105, 663-680, 2000.

Larson, K. M., J. T. Freymueller, and S. Philipsen, Global plate velocities from the Global Positioning System, J. Geophys. Res., 102, 9961-9981, 1997.

Minster, J. B. and T. H. Jordan, Present-day plate motions, J. Geophys. Res., 83, 5331-5354, 1978.

Norabuena, E. O., T. H. Dixon, S. Stein, and C. G. A. Harrison, Decelerating Nazca-South America and Nazca-Pacific plate motions, Geophys. Res. Lett., 26, 3405-3408, 1999.

Shen, Z.-K., C. Zhao, A. Yin, Y. Li, D. D. Jackson, P. Fang, and D. Dong, Contemporary crustal deformation in east Asia constrained by Global Positioning System measurements, J. Geophys. Res., 105, 5721-5734, 2000.

Shen-Tu, B., W. E. Holt, and A. J. Haines, Contemporary kinematics of the western United States determined from earthquake moment tensors, very long baseline interferometry, and GPS observations, J. Geophys. Res., 103, 18,087-18,117, 1998.

Shen-Tu, B., W. E. Holt, and A. J. Haines, The kinematics of the western United States estimated from Quaternary fault slip rates and recent geodetic data, J. Geophys. Res., 104, 28,927-28,955, 1999.

Spitzak, S. and C. DeMets, Constraints on present-day plate motions south of $30^{\circ} \mathrm{S}$ from satellite altimetry, Tectonophysics, 253, 167-208, 1996.

Stein, S., Space geodesy and plate motions, in Contributions of Space Geodesy to Geodynamics: Crustal Dynamics, Geodyn. Ser., 23, edited by D. E. Smith and D. L. Turcotte, pp. 5-10, AGU, Washington, D.C., 1993.

Zumberge, J. F., Liu, R., (eds.), Densification of the IERS Terrestrial Reference Frame through regional GPS networks, IGS workshop proc., Pasadena, California, IAG/IUGG, 1995.

C. Kreemer (e-mail: kreemer@horizon.ess.sunysb.edu), J. Haines, W. E. Holt, G. Blewitt, and D. Lavallee 